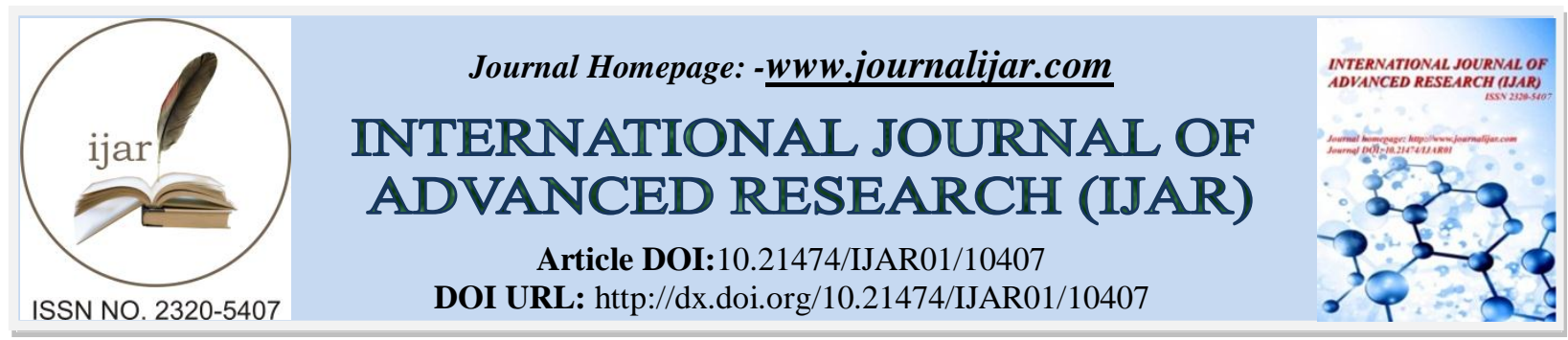

RESEARCH ARTICLE

\title{
CORRELATION OF ANTHRACYCLINE INDUCED EARLY ONSET CHRONIC CARDIO TOXICITY WITH PRO BNP AND ECHOCARDIOGRAPHY
}

\author{
Dr. Asif Riaz Khan, Dr. Fauzia Abdus Samad, Dr. Abdus Samad, Dr. Sharmin Arif and Dr. Anum Khan \\ Fauji Foundation Hospital Rawalpindi, Pakistan.
}

\section{Manuscript Info}

Manuscript History

Received: 30 November 2019

Final Accepted: 31 December 2019

Published: January 2020

\section{Abstract}

\section{Introduction:-}

During the last few decades, overall survival of cancer patients has improved. Now more cancer survivors reach old age, in which cardiovascular diseases remain the leading cause of mortality. ${ }^{[1]}$ Cardio toxicity secondary to chemotherapy also puts cancer survivors at high risk of death secondary to cardiovascular events. ${ }^{[2]}$ Anthracyclines (Doxorubicin, Daunorubicin, Idarubicin, Epirubicin and Mitoxantrone) are the class of chemotherapeutic agents that are potentially cardio toxic. ${ }^{[3]}$ Cardio toxicity caused by anthracyclines can present as acute and chronic cardiotoxicity. Acute cardiotoxicity is seen in $<1 \%$ of the patients which is usually reversible and self-limiting. ${ }^{[4]}$ Chronic toxicity however can have two presentations, either as early onset chronic toxicity and or late onset chronic toxicity. ${ }^{[5]}$ Early onset chronic cardio toxicity happens within first 12 months of chemotherapy, with peak incidence documented in the initial three months. ${ }^{[6]}$ Late onset cardio toxicity develops after 12 months of chemotherapy and can manifest as late as 1-2 decades after chemotherapy. The late onset cardio toxicities are not reversible. ${ }^{[4]}$

Anthracycline are assumed to cause cardiomyocyte impairment by forming free radicles and reactive oxygen species which contribute to oxidative stress resulting in mitochondrial damage and cell death ${ }^{[7]}$. Moreover, calcium and iron hemostasis is also affected by anthracycline which contribute to cardiomyocyte damage. Topoisomerase $2 \beta$ is another mechanism of causing injury to non-proliferative cells like cardiomyocyte. ${ }^{[7][8]}$ These different mechanisms are thought to be causing the cardiac cell death.

Early detection of cardio toxicity in patients treated with anthracyclines remains an important topic for research. Different biomarkers like troponins, brain natriuretic peptide (BNP), C reactive protein (CRP), myeloperoxidase (MPO), galectin-3 and nitrous oxide have been studied for detecting cardiotoxicity caused by anthracyclines. ${ }^{[3][6][9][10]}$ Azher et al reported high levels of Troponin I in patients who developed cardiotoxicity after Anthracycline based chemotherapy. ${ }^{[6]}$ Lipshultz et al found high CRP values in pediatric cancer survivors. In addition to these studied biomarkers certain other markers are also under evaluation and have been evaluated in a couple of studies. Myeloperoxidase is pro inflammatory enzyme. Ky et al found that high MPO levels were related to risk of cardiotoxicity after anthracycline based chemo therapeutic agents at 15 months follow up. Nitrous oxide is another marker that plays a vital role in cardiomyocyte contractility. Guler at al noticed high nitrous oxide values in pediatric patients who received with doxorubicin with post chemotherapy low left ventricular ejection fraction (LVEF) and fractional shortening. ${ }^{[9]}$ A correlational analysis by Annop et al noticed that high pro b-type natriuretic peptide (ProBNP) levels were associated with fractional shortening. ${ }^{[3]}$ 
BNP is produced in ventricles in response to increased wall stress and is being widely studied globally. It is converted to ProBNP which is further converted to NT Pro BNP. ${ }^{[3]}$ Persistent rise in BNP is an indication of LV wall stress, diastolic pressure and volume overload. High ProBNP reflects LVEF reduction and its levels have linear relationship to the severity of heart failure. ${ }^{[9]}$ BNP and NT ProBNP are better predictors of cardiotoxicity in asymptomatic patients treated with anthracycline due to its long half-life. ${ }^{[1]}$ Echocardiography is non-invasive and easily available investigation which can detect cardiotoxicity induced by chemotherapeutic agents. ${ }^{[5]}$ LVEF measured by echo is the current standard of care for monitoring cardiotoxicity secondary to chemotherapeutic agents. ${ }^{[12][13]}$

The aim of this study was to correlate the rise in ProBNP with echocardiographic findings in patients who received anthracycline based chemotherapy. Identifying marker which can predict anthracycline related cardio toxicity will help to detect patients at high risk of cardiac failure so that early interventions can be implemented to reduce cardio toxicity related mortality.

\section{Objective:-}

The Objective of this study was:

1. To detect a pattern of rise in Pro BNP in response to anthracycline based chemotherapies

2. To see relationship of Pro BNP with early onset anthracycline related cardio toxicity in asymptomatic patients receiving anthracycline based chemotherapies at Oncology department FFH.

\section{Operational Definition:-}

1. Cardio toxicity:- It is asymptomatic decrease of $10-15 \%$ in LVEF within normal range or decrease in LVEF below the normal range. ${ }^{[2]}$

2. Pro BNP:- Pro BNP is natriuretic peptide secreted by ventricles in response to LV wall stress. The upper normal limit is $<125 \mathrm{pg} / \mathrm{ml}^{[14]}$

\section{Material and Methods:-}

It was an observational case series with a quantitative correlational study design. Study was conducted at Oncology Department, Fauji Foundation Hospital, Rawalpindi.

Total duration of study was 15 months starting from date of approval. A total of 57 patients were included based on parent study data.

Patients were enrolled in the study using consecutive non probability sampling method. Inclusion Criteria included all those:-

1. Patient aged $\geq 12$ years

2. Histopathological evidence of malignancy requiring anthracycline based chemotherapy

3. Baseline echocardiography showing Ejection Fraction $>50 \%$

4. Normal Blood Counts, Liver and kidney function tests.

All those patients with below mentioned conditions were excluded from the study:-

1. Patients who received previous Chemotherapy.

2. History of cardiac conditions like coronary artery disease, Congestive heart failure, Arrhythmias, Valvular heart diseases, Chronic obstructive pulmonary disease

3. Hypertension with end organ damage

4. Patients taking ACE inhibitors.

5. Diabetes with end organ damage

6. Pregnancy

In this study, patients with histologically proven malignancy were recruited from Oncology out-patient at Fauji Foundation Hospital,Rawalpindi. After taking an informed consent, selection of patients was carried out on nonprobability consecutive sampling technique. After enrolling the patient in study, detailed history, examination, blood $\mathrm{CP}$, renal and liver function tests and staging work up was done. Echocardiography and Pro BNP were done before starting chemotherapy. Echocardiogram was repeated at 52 weeks after the first chemotherapy cycle. ProBNP levels 
were repeated at 3 weeks, 12 weeks and 52 weeks after the first chemotherapy cycle to assess for any change in its levels as well as the final correlation with the echocardiogram.

SPSS version 23 was used to analyze the data. Descriptive statistics included mean and standard deviations for continuous variables like age and Pro BNP values. Frequencies were calculated for categorical variables like tumor types and chemotherapy regimens used. The trend of ProBNP change was monitored from baseline till week 52. Also, the ProBNP values were correlated with the echocardiographic change in ejection fraction at week 52, to assess for long term cardiotoxicity.

\section{Data collection procedure:-}
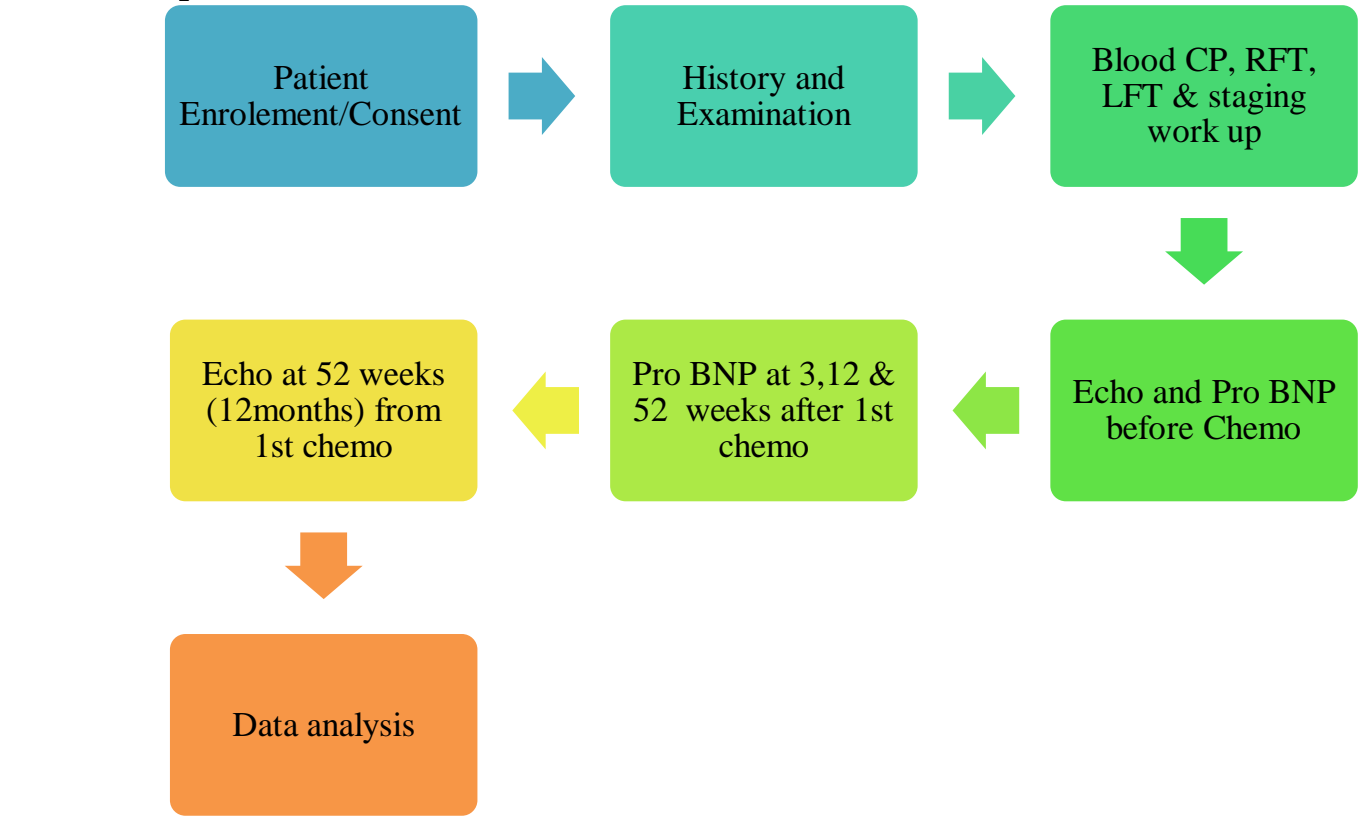

\section{Results:-}

In this study a total of 57 patients with histological proven malignant disorders were enrolled. The mean age of patients was $46.1 \pm 14.8$ years and most of them (38 patients) were between 41 to 60 years of age. In our study population, 53 patients $(93.0 \%)$ were females and 4 patients $(7 \%)$ were males. Forty nine percent females were premenopausal and $51 \%$ females were post-menopausal (Table 1).

Breast carcinoma was the most common diagnosis $(81.0 \%)$ followed by acute lymphocytic leukemia $(9.0 \%)$, acute myeloid leukemia (7.0\%) and Hodgkin's lymphoma (3.0\%) as shown in Figure I.

Most commonly used chemotherapy regimens were, FEC (Farmarubicin, epirubicin, cyclophosphamide) regimen, used in 28 patients (49.1\%) and AC(Adriamycin, cyclophosphamide) $x$ IV $\rightarrow$ Paclitaxel $x$ IV regimen, prescribed to 12 patients $(21.1 \%)$. The other frequent regimens were UKALL taken by 5 patients $(8.8 \%)$, D3A7 (Daunorubicin, Ara-C) used in 3 patients $(5.3 \%)$ and FAC (Farmarubicin, adriamyicin, cyclophosphamide), AT (Adriamycin, paclitaxel) and $\mathrm{ABVD}$ (Adriamycin, bleomycin, vinblastine, dacarbazine) given to 2 patients each(3.5\%), See table 2 for chemotherapies details.

In descriptive statistics, ProBNP values were expressed as mean and standard deviations, as well as medians due to heterogeneity of data. There is a gradual decrease in the mean ProBNP levels at baseline from 105.4 to 93.2 at 3 weeks and then 74.1 at 12 weeks. However, after that it showed a rise in mean ProBNP at 52 weeks to 141.9 as shown in table 3.

The aim of this study was to see any correlation between ProBNP levels and cardiotoxicity in the study patients. However, in this study 52 weeks after chemotherapy none of the patients developed cardiotoxicity. There were 8 patients who died due to various complications not related to cardiotoxicity. Amongst the deceased patients, four leukemia patients died due to failure to achieve remission, two breast cancer patients developed brain metastasis and 
died secondary to it, one patient passed away after developing Guillan-Barre Syndrome and one died due to febrile neutropenia.

\begin{tabular}{|l|l|l|}
\hline Age (years) & No. of cases & \%age \\
\hline Up to 20 & 7 & $12.3 \%$ \\
\hline 21 to 40 & 4 & $7.0 \%$ \\
\hline 41 to 60 & 38 & $66.7 \%$ \\
\hline 61 or above & 8 & $14.0 \%$ \\
\hline Premenopausal females & 26 & $49 \%$ \\
\hline Postmenopausal females & 27 & $51 \%$ \\
\hline Mean \pm SD & $46.1 \pm 14.8$ & \\
\hline Median & 49.0 & \\
\hline Gender & & $7.0 \%$ \\
\hline Male & 4 & $93.0 \%$ \\
\hline Female & 53 & \\
\hline
\end{tabular}

Table 1:- Demographic and clinical characteristics of patients.

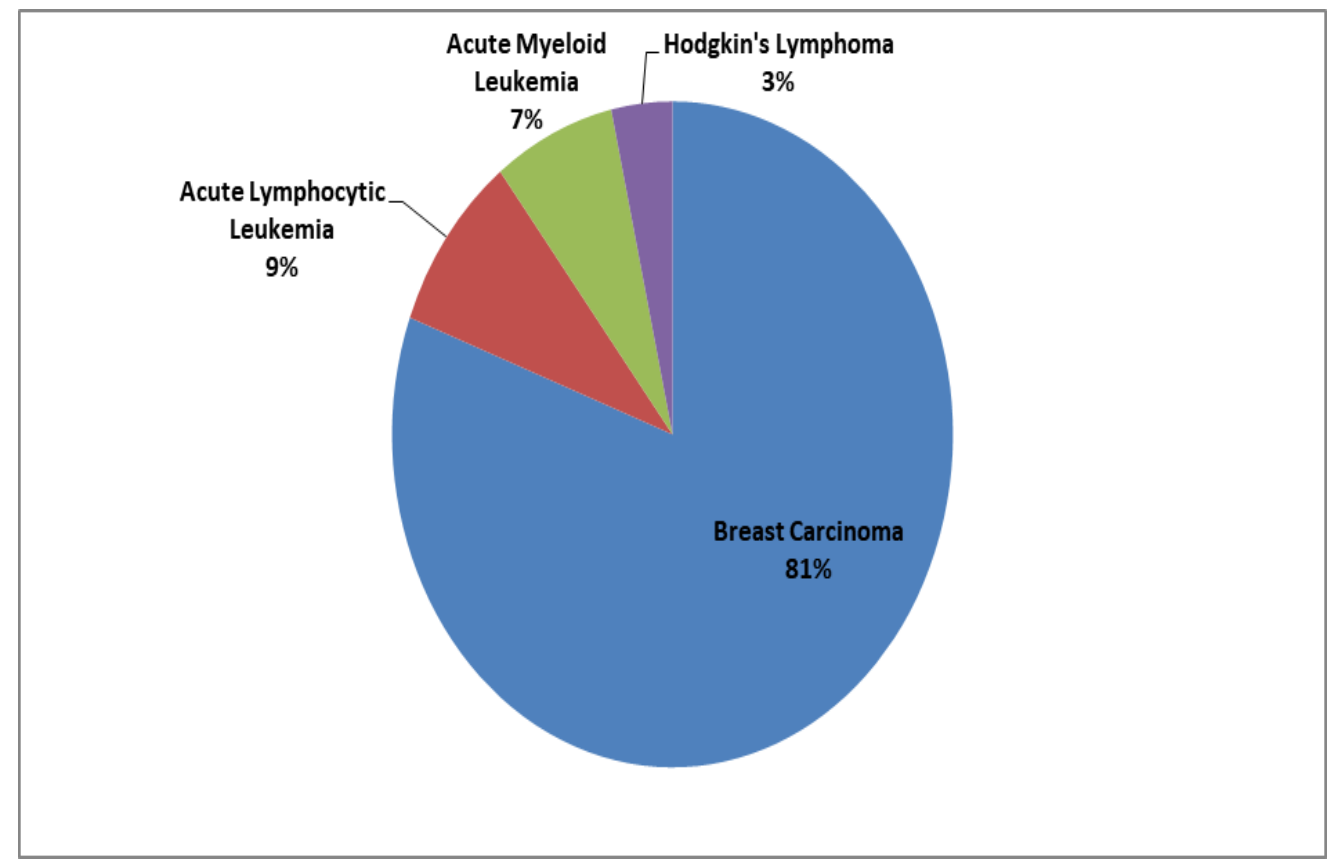

Figure 1:- Diagnosis of study patients $(n=57)$.

\begin{tabular}{|l|l|l|}
\hline Chemotherapy Regimen & Frequency & Percent \\
\hline FEC(Farmarubicin,epirubicin,cyclophosphamide) x VI & 28 & $49.1 \%$ \\
\hline AC( Adriamycin,cyclophosphamide) x IV $\rightarrow$ Pacli x IV & $21.1 \%$ \\
\hline UKALL XI & 12 & $8.8 \%$ \\
\hline D3A7 ( Daunorubicine, cytarabine) & 5 & $5.3 \%$ \\
\hline FAC(Farmarubicin,adriamycin,cyclophosphamide x VI & 3 & $3.5 \%$ \\
\hline AT (Adriamycin , Paclitaxel) & 2 & $3.5 \%$ \\
\hline ABVD(Adriamycin,bleomycin,vinblastine,dacarbazine) & $3.5 \%$ \\
\hline Atra plus Idarubicin & 2 & $1.8 \%$ \\
\hline EC( epirubicin, cyclophosphamide) x IV $\rightarrow$ Pacli x IV & 2 & $1.8 \%$ \\
\hline TAC (Docetaxel, doxorubicin, cyclophosphamide) & 1 & $1.8 \%$ \\
\hline
\end{tabular}

Table 2:- Chemotherapy regimen given to patients $(n=57)$.

\section{ProBNP findings}

At baseline

\section{Mean \pm SD}

$105.4 \pm 147.7$ 


\begin{tabular}{|l|l|}
\hline At 3 weeks & $93.2 \pm 160.8$ \\
\hline At 12 weeks & $74.1 \pm 126.8$ \\
\hline At 52 weeks & $141.9 \pm 760.4$ \\
\hline
\end{tabular}

Table 3:- Average findings on ProBNP investigation at different follow-ups $(n=57)$.

\section{Discussion:-}

Cancer survival rate has improved to $67 \%$ for patients above the age of 65 years owing to improvement in treatment modalities. ${ }^{[10]}$ Chemotherapy induced cardiotoxicity, hence has attracted attention of both the oncologist and the cardiologist alike. Accomplishments of chemotherapy are annulled by their potential undesired cardio toxic effects. ${ }^{[15]}$ Anthracyclines are highly active antineoplastic agents used in various hematological malignancies and solid tumors. ${ }^{[16][17]}$ Anthracycline induced cardio toxicity has been extensively studied with mechanism of cardiotoxicity not yet fully understood, though various hypothesis have been postulated. ${ }^{[18]}$

Prevention and early detection of anthracycline induced cardiotoxicity seems to be the way forward in mitigating the life threatening and irreversible adverse effect of anthracyclines. To date, no established guidelines recommend screening for early recognition of anthracycline induced cardio toxicity ${ }^{[19]}$.

In our study, 49 patients were below the age of 60 years with mean age of 46.1 years. There were $93 \%$ female patients and males being $7 \%$ in our study population, as our hospital is looking after the families of veterans. Almost half $(49 \%)$ of the study population patients were premenopausal. Skovgaurd et al reported positive correlation of ProBNP and cardiotoxicity secondary to anthracyclines ${ }^{[2]}$. Their study population included 333 patients with mean age of 49.1 years and all patients being females with a follow up of 6 years ${ }^{[2]}$. Despite the similarity in age and gender, the absence of cardiotoxicity in our study, possibly could be due to shorter follow up and smaller cohort size in our study. Sawaya et al did not find any association between ProBNP and cardiotoxicity. Their study population had 43 patients with mean age of 47 years. All the patients were females with a follow up period of 6 months ${ }^{[14]}$. So this has guided us to continue monitoring our study population after the study time to identify any significant cardiotoxicity over long period.

In our study breast cancer was the most common histology (81\% of patients) followed by acute lymphocytic leukemia, acute myeloid leukemia and Hodgkin's lymphoma respectively. This was quite similar to the study population of Skovgaurd et al. ${ }^{[2]}$ Sawaya et al included patients with carcinoma of breast and they could not find any association between change in ProBNP and cardiotoxicity. ${ }^{[1]}$ This again could be explained by follow up duration difference between the two studies.

Important finding in our study was the well tolerated chemotherapy regimens without any adverse cardiac outcomes which can be an important area to explore in future. This also could be explained by different genetic makeup of our population which can be contributing to better tolerability of anthracyclines by our patients. Patients of Asia Pacific region exhibit different behavior when compared to western counterparts. ${ }^{[20]}$

Our study population was from lower socioeconomic class who has less sedentary lifestyle as compared to western countries. Due to cultural and social reason, smoking and alcohol use in also almost nonexistent which all could contribute to better tolerability of cardio-toxic drugs.

However, mean ProBNP levels at week 52 showed a rise after the steady initial decline which might be the start of anthracycline induced delayed cardiotoxicity. Though the echocardiography at week 52 showed normal ejection fraction in all patients who completed and no clinically overt cardiotoxicity in patient, this rise in ProBNP could be the harbinger of delayed onset cardiotoxicity. This can also guide us that that ProBNP may precede the onset of cardio-toxic symptoms quite before the echocardiography can detect it. However, this assumption cannot be fully defended till these patients are followed longer enough to develop cardiotoxicity which is detected by clinical manifestation or echocardiographic findings. Because we have these established pro-symptomatic significance of serological markers in ovary, prostate and gastrointestinal tract tumors, where we use serum cancer antigen 125 (CA125),Prostate-specific antigen (PSA) and Carcinoembryonic antigen (CEA) levels to detect the serological relapse at times quite before the radiological presentation of these diseases ${ }^{[21]}$. 
Our study had few limitations. We had used non-probability sampling and sample size as well as study duration were our main limiting factors. Large randomized multi-centric studies with longer follow up might detect late onset cardiotoxicity in these patients. There are other causes of high BNP which might have accounted for raised Pro BNP levels at base line. Elderly patients and females can have high normal natriuretic peptide values. ${ }^{\text {[2] }}$. In our study 53 patients were females and 4 were males. This could also be the reason of high normal values of ProBNP in patients who had slightly raised ProBNP. Malignancy by itself can cause high ProBNP level. Patients with metastatic disease have higher levels or ProBNP than patients with non-metastatic disease. ${ }^{[22][23]}$ In our study, 7 patients $(12.2 \%)$ had stage IV disease.

Though we did not find any positive correlation between rise in ProBNP and cardiotoxicity caused by anthracycline, but the late rise of ProBNP levels might be really significant finding in our population which can guide us to plan and design further larger and longer duration studies. We propose follow up studies which can look into existing and new markers of cardiotoxicity with different monitoring schedules and longer follow ups. It will be worthwhile to explore new markers to try finding biomarkers which can detect anthracycline induced cardio toxicity early in its treatment course to help reduce the morbidity and mortality associated with this class of drug.

\section{References:-}

1. Curigliano G, Cardinale D, Dent S, Criscitiello C, Aseyev O, Lenihan D, et al. Cardiotoxicity of anticancer treatments: Epidemiology, detection, and management. CA Cancer J Clin [Internet] 2016;66(4):309-25. Available from: doi: 10.3322/caac.21341.

2. Skovgaard D, Hasbak P, Kjaer A. BNP Predicts Chemotherapy-Related Cardiotoxicity and Death : Comparison with Gated Equilibrium Radionuclide Ventriculography. PLoS One [Internet] 2014;9(5):1-10. Available from: https://www.ncbi.nlm.nih.gov/pmc/articles/PMC4011788/

3. Annop KITTIWARAWUT 1, Yongkasem VORASETTAKARNKIJ 2, Suebpong TANASANVIMON 1, Sopark MANASNAYAKORN3 4, And, SRIURANPONG V. Serum NT-proBNP in the early detection of doxorubicin-induced cardiac dysfunction Serum NT-proBNP in the early detection of doxorubicin-induced cardiac dysfunction. Asia Pac J Clin Oncol [Internet] 2013;9(August 2012):155-61. Available from: https://www.researchgate.net/publication/230684416

4. Rosa GM, Gigli L, Tagliasacchi MI, Di Iorio C, Carbone F, Nencioni A, et al. Update on cardiotoxicity of anticancer treatments. Eur. J. Clin. Invest.2016;46(3):264-84.

5. nosheen fatima. CARDIOTOXICITY OF ANTICANCER DRUGS. Pakistan J Radiol [Internet] 2009;19(1):147. Available from: http://www.pakjr.com/ojs/index.php/PJR/article/view/349

6. Shafi A, Siddiqui N, Imtiaz S, Ud M, Sajid D. LEFT VENTRICULAR SYSTOLIC DYSFUNCTION PREDICTED BY EARLY TROPONIN I RELEASE AFTER ANTHRACYCLINE BASED CHEMOTHERAPY IN BREAST CANCER PATIENTS. J Ayub Med Coll Abbottabad-Pakistan [Internet] 2017;29(2):266-9. Available from: http://jamc.ayubmed.edu.pk/index.php/jamc/article/view/2842

7. Lewalle A, Land S, Merken JJ, Raafs A, Sepúlveda P, Heymans S, et al. Balance of Active, Passive, and Anatomical Cardiac Properties in Doxorubicin-Induced Heart Failure. Biophys J 2019;1-12.

8. Cai F, Luis M, Lin X, Wang M, Cai L, Cen C, et al. Anthracycline-induced cardiotoxicity in the chemotherapy treatment of breast cancer: Preventive strategies and treatment (Review). Mol Clin Oncol 2019;15-23.

9. Tian S, Hirshfield KM, Jabbour SK, Toppmeyer D, Haffty BG, Khan AJ, et al. Serum Biomarkers for the Detection of Cardiac Toxicity after Chemotherapy and Radiation Therapy in Breast Cancer Patients. Front Oncol [Internet] 2014;4(October):1-14. Available from: https://doi.org/10.3389/fonc.2014.00277

10. Moazeni S, Cadeiras M, Yang EH, Deng MC, Nguyen K-L. Anthracycline induced cardiotoxicity: biomarkers and "Omics" technology in the era of patient specific care. Clin Transl Med 2017;6(1).

11. Cardinale D, Sandri MT. Detection and monitoring of cardiotoxicity by using biomarkers: Pros and cons: Remarks on the international colloquium on cardioncology. [Internet]. Prog. Pediatr. Cardiol.2015;39(2):77-84. Available from: http://dx.doi.org/10.1016/j.ppedcard.2015.10.004

12. Pizzino F, Vizzari G, Bomzer CA, Qamar R, Carerj S, Zito C, et al. Diagnosis of Chemotherapy-Induced Cardiotoxicity. J Patient-Centered Res Rev [Internet] 2014;1(3):121-7. Available from: http://digitalrepository.aurorahealthcare.org/jpcrr/vol1/iss3/3

13. Tadic M, Cuspidi C. The Role of Echocardiography in Detection of Chemotherapy-Induced Cardiotoxicity in Breast Cancer Patients. Int J Cancer Manag [Internet] 2017;10(5):e8109. Available from: http://ijcancerprevention.com/en/articles/8109.html 
14. Sawaya H, Sebag IA, Plana JC, Januzzi JL, Ky B, Cohen V, et al. Early Detection and Prediction of Cardiotoxicity in Chemotherapy-Treated Patients. Am J Cardiol [Internet] 2011;107(9):1375-80. Available from: https://linkinghub.elsevier.com/retrieve/pii/S000291491100141X

15. Corremans R, Adão R, De Keulenaer GW, Leite-Moreira AF, Brás-Silva C. Update on pathophysiology and preventive strategies of anthracycline-induced cardiotoxicity. Clin Exp Pharmacol Physiol 2019;46(3):204-15.

16. Bunney PE, Zink AN, Holm AA, Billington CJ, Kotz CM. Orexin activation counteracts decreases in nonexercise activity thermogenesis (NEAT) caused by high-fat diet. Physiol Behav [Internet] 2017;176(3):13948. Available from: https://linkinghub.elsevier.com/retrieve/pii/S0031938416312148

17. Zidan A, Sherief LM, El-Sheikh A, Saleh SH, Shahbah DA, Kamal NM, et al. NT-proBNP as early marker of subclinical late cardiotoxicity after doxorubicin therapy and mediastinal irradiation in childhood cancer survivors. Dis Markers 2015;2015.

18. Wenningmann N, Knapp M, Ande A, Vaidya TR, Ait-Oudhia S. Insights into Doxorubicin-induced Cardiotoxicity: Molecular Mechanisms, Preventive Strategies, and Early Monitoring. Mol Pharmacol 2019;96(2):219-32.

19. Ganame J, Jurcut R, Wildiers H, Ganame J. Detection and monitoring of cardiotoxicity - What does modern cardiology offer? Detection and monitoring of cardiotoxicity — what does modern cardiology offer? Support Cancer Care 2008;16(June):437-45.

20. Rajadurai J, Tse HF, Wang CH, Yang NI, Zhou J, Sim D. Understanding the Epidemiology of Heart Failure to Improve Management Practices: An Asia-Pacific Perspective. J Card Fail [Internet] 2017;23(4):327-39. Available from: http://dx.doi.org/10.1016/j.cardfail.2017.01.004

21. Vaidyanathan K, Vasudevan DM. Organ specific tumor markers: What's new? Indian J Clin Biochem 2012;27(2):110-20.

22. Tan LL, Lyon AR. Role of Biomarkers in Prediction of Cardiotoxicity During Cancer Treatment. Curr Treat Options Cardiovasc Med 2018;20(7).

23. Bando S, Soeki T, Matsuura T, Tobiume T, Ise T, Kusunose K, et al. Plasma brain natriuretic peptide levels are elevated in patients with cancer. PLoS One 2017;12(6):1-15. 